Article

\title{
Multi-Criteria Decision Making for Efficient Tiling Path Planning in a Tetris-Inspired Self-Reconfigurable Cleaning Robot
}

\author{
Maryam Kouzehgar 1,*(D), Mohan Rajesh Elara ${ }^{1}$, Mahima Ann Philip ${ }^{1}$, \\ Manimuthu Arunmozhi ${ }^{1,2}$ and Veerajagadheswar Prabakaran ${ }^{1}$ \\ 1 Engineering Production and Development Pillar, Singapore University of Technology and Design, \\ Singapore 487372, Singapore; rajeshelara@sutd.edu.sg (M.R.E.); philpsmahima@gmail.com (M.A.P.); \\ maniasaldhinesh@gmail.com (M.A.); prabakaran@sutd.edu.sg (V.P.) \\ 2 College of Engineering Guindy, Anna University, Chennai 600025, India \\ * Correspondence: maryam_kouzehgar@sutd.edu.sg; Tel.: +65-8776-9026
}

Received: 26 September 2018; Accepted: 21 December 2018; Published: 25 December 2018

\begin{abstract}
In this study, we aim to optimize and improve the efficiency of a Tetris-inspired reconfigurable cleaning robot. Multi-criteria decision making (MCDM) is utilized as a powerful tool to target this aim by introducing the best solution among others in terms of lower energy consumption and greater area coverage. Regarding the Tetris-inspired structure, polyomino tiling theory is utilized to generate tiling path-planning maps which are evaluated via MCDM to seek a solution that can deliver the best balance between the two mentioned key issues; energy and area coverage. In order to obtain a tiling area that better meets the requirements of polyomino tiling theorems, first, the whole area is decomposed into five smaller sub-areas based on furniture layout. Afterward, four tetromino tiling theorems are applied to each sub-area to give the tiling sets that govern the robot navigation strategy in terms of shape-shifting tiles. Then, the area coverage and energy consumption are calculated and eventually, these key values are considered as the decision criteria in a MCDM process to select the best tiling set in each sub-area, and following the aggregation of best tiling path-plannings, the robot navigation is oriented towards efficiency and improved optimality. Also, for each sub-area, a preference order for the tiling sets is put forward. Based on simulation results, the tiling theorem that can best serve all sub-areas turns out to be the same. Moreover, a comparison between a fixed-morphology mechanism with the current approach further advocates the proposed technique.
\end{abstract}

Keywords: self-reconfigurable robot; cleaning robot; Tetris-inspired; polyomino tiling theory; coverage path planning; area decomposition; multi-criteria decision making

\section{Introduction}

Robots are deployed in a wide range of applications and are, therefore, rapidly becoming an integral component of our everyday life. In particular, with several million units sold worldwide, robots developed and implemented for floor cleaning tasks will become an elemental part of the household in the near future. Such a massive market in robotic floor cleaning products involves dominant market players such as IRobot, Dyson, Samsung, Xiaomi, and Neato. These robots can autonomously navigate through a given area using onboard sensors and are typically characterized by circular, square, and D-shaped morphologies. The suitability of current robots to assist in daily domestic tasks is investigated in [1] from the human user viewpoint. Additionally, the study presented in [2], analyses and models floor-cleaning coverage performances of some commercial domestic 
mobile robots. Moreover, not only in the commercial market but also in the academic literature, there exist numerous cleaning robot studies within a wide spectrum from mechanical design to autonomy. A Swedish wheeled mechanism for a floor cleaning robot was presented in [3] in order to attain efficient locomotion in a populous area. In terms of robot autonomy, an innovative neural network approach is presented in [4] which is utilized for path planning and obstacle avoidance for a cleaning robot in a random environment. On the other hand, since numerous robotic floor cleaning products are available in the marketplace, it is critical to have benchmark schemes to validate the robot's cleaning performance and coverage efficacy [5]. In addition, multi-robot schemes have been proved to be useful in cleaning applications [6]. Although there are numerous studies that highlight the benefits of cleaning robots, the efficiency of traditional cleaning robots is still affected by factors such as fixed morphology. Since such a rigid structure highly limits the accessible spaces like room corners and narrow corridors, one viable approach to overcome this restriction in a traditional cleaning system is to develop next-generation robots with shape-shifting abilities to maximize the area coverage performance.

So far, in the field of reconfigurable robotics, three different architectures are introduced, namely, intra-reconfiguration, inter-reconfiguration, and nested reconfiguration. Intra-reconfiguration deals with a single robotic system that could change its morphology on its own without any external supports [7]. Robots proposed under the inter-reconfigurable principle are basically modular robots that can possess different morphologies by undergoing assembling and disassembling processes [8]. Modular robots can be investigated at higher levels to benefit from swarm intelligence. In this regard, modular swarm robots are utilized to gain advantages on self-reconfigurability, self-replication, and self-assembly [9]. On the other hand, nested reconfiguration can perform both inter and intra reconfigurations. Hinged Tetro presented in [10], is an example of such nested reconfigurable robots. Although numerous studies in the literature address reconfigurable robotics, they are primarily limited to mechanism design and are hardly implemented to an area coverage task like floor cleaning. In order to bridge this gap, in our previous work, we presented a novel reconfigurable floor cleaning robot called hTetro [11,12]. This Tetris-inspired structure can change its morphology to any of the one-sided Tetris pieces and as a result, could achieve superior coverage performance through its shapeshifting ability. In addition, in a couple of our previous works, we utilized polyomino tiling theory to introduce a coverage path planning technique for Tetris-inspired polyomino robots [13,14].

Polyomino tiling theory has been extensively studied in combinatorial mathematics as a distinct branch of mathematics with Golomb as the pioneering inventor in this field. The applications of this theory span a wide variety of fields including medicine [15], graphics, and gaming [16]. However, polyomino tiling theory has rarely been applied to the field of robotics and, therefore, presents a scope for tremendous research. As briefly mentioned above, in [13,14], a novel approach is introduced to harness the polyomino tiling theory as a way of suggesting navigation strategies for reconfigurable cleaning robots. These tiling theorems equip the reconfigurable robots with superlative navigation abilities and make corners and narrow spaces more accessible. In pursuance of [14], this paper utilizes the polyomino tiling theory to propose tiling sets as navigation strategies of the Tetris-inspired cleaning robot in a static environment but goes a step further to definitively select the optimal tiling set for each area under cleaning. This will suggest the navigation strategy that improves the overall efficiency from the viewpoint of area and energy. This way, we will be entering the borders of optimal navigation for a Tetris-inspired reconfigurable cleaning robot.

In our previous experiments on shape-shifting cleaning robots, we only considered area coverage as a single parameter for assessing the robot's performance, which is actually insufficient when the robot is functioning in real-time scenarios [13,14]. Since the amount of energy consumption is also a significant issue in any electromechanical system, the current paper aims at balancing a trade-off between energy consumption and area coverage. Thus, in this study, we consider these two key factors as decision criteria in a Multi-Criteria Decision Making (MCDM) process in order to choose the best navigation strategy (defined by the tiling set) for achieving higher efficiency in terms of less energy with superior area coverage. 
MCDM is a general term referring to all methods that help to make decisions based on preferences where there is more than one conflicting criterion [17]. The reason why we apply MCDM is that generally, real applications require the consideration of several possibly conflicting objectives to be fulfilled, thus a multi-objective problem is required to be solved. On the other hand, usually a trade-off between the objectives exists and we rarely have an alternative that can best satisfy all the objectives simultaneously. Accordingly, the intelligent agent must efficiently balance the facing trade-off.

Despite the fact that MCDM methods may be widely diverse, many of them share certain aspects in common [18], such as the concepts of alternatives, criteria, and the weights of importance.

- Alternatives represent the different choices available to the decision maker. Usually, the set of alternatives is assumed to be finite.

- Criteria represent the different factors on the basis of which the alternatives can be investigated.

- Importance factors (weights of importance) are considered as a measure of the significance of each criterion in the decision-making process. Usually, these weights are normalized to add up to one. It is also assumed that the decision maker has determined the weights of the decision criteria based on relative significance.

If the number of alternatives is infinite, then we will face a multi-objective optimization (MOO). Here, since we have a finite set of tiling sets proposed based on the tiling theorems, we are supposed to solve a MCDM regarding the area coverage and energy as the decision-making criteria. Moreover, there are many ways to classify MCDM methods:

- Classification based on the data type: deterministic, stochastic, or fuzzy MCDM methods [19]. However, some situations require combinations of all the above [20].

- Classification based on the number of decision makers: Single vs. group MCDM [21].

- Classification based on the approach applied on the data: The WSM (Weighted Sum Model) or SAW (Simple Additive Weighting), WPM (Weighted Product Model), AHP (Analytic Hierarchy Process), revised AHP, ANP (Analytic Network Process), TOPSIS (Technique for Order Preference by Similarity to Ideal Solution), ELECTRE (Elimination and Choice Translating Reality), PROMETHEE (Preference Ranking Organization Method for Enrichment Evaluations); VIKOR (VlseKriterijumska Optimizacija I Kompromisno Resenje), and LINMAP (Linear Programming for Multidimensional Analysis of Preference) are the methods which are of more use in practice $[17,18,22]$.

In addition, MCDM plays a significant role in many intelligent systems and is widely utilized in many fields such as operation research and soft computing [21,23]. Moreover, regarding the links between MCDM and robotics, it is mostly utilized for the allocation of resources and task scheduling in robotic swarms [24], robot selection in automated industry operations [25], and defining exploration strategies for search and rescue robots [26]. Similarly, in this paper, MCDM will be applied to choose between navigation strategies defined by given tiling sets. Here, we will be focused on single decision-making deterministic MCDM applying concepts of the SAW (Simple Additive Weighting) method [22].

In summary, all aspects of this research are quite novel. In better words, all aspects of it have recently emerged: The mechanical design of the Tetris-inspired cleaning robot is proposed in 2017 [11,12] and the path-planning idea is raised in 2018 in terms of a tiling theory approach [13,14]. However, the previously raised tiling-path planning approach only considers area coverage as a single performance metric with absolutely no consideration of energy consumption issues. The main contributions of this study in comparison to our previous works are as follows:

- In this study, energy consumption is considered as a second significant performance metric in tiling path planning.

- This paper proposes an energy estimation scheme for the novel self-reconfigurable robotic platform. 
- The current work suggests the optimal path-planning approach by means of MCDM, i.e., creating an optimal balance between maximum area coverage and minimum energy. In other words, however the tiling-theoretic path planning approach is introduced in [13,14], this paper goes beyond previous works to some degree by taking into account the energy factor and aims at adding some optimality utilizing MCDM.

- In addition, in this study, a more recent tiling theorem has been utilized that is not investigated in previous studies $[13,14]$ and surprisingly, based on simulation results, this specific theorem (here illustrated as Theorem 1 [27]) turns out to be the most-promising tiling theorem that can best serve all the areas targeted for optimal cleaning.

On the whole, tiling-path-planning is a novel concept only related to the novel hTetro, and it is our belief that expanding a novel idea (by considering more parameters) and adding optimality to a novel concept is invaluable.

The rest of the paper is organized as follows. Section 2 introduces the experimental environment and explains the utilized Tetris-inspired robot structure. Section 3 casts light on the concepts of Polyomino Tiling Theory applied to our robotic platform and elaborates the four utilized tiling theorems. Moreover, this section is also concerned with the reason (and also technique) for the area decomposition process and finally presents five distinguished tiling sets under each theorem applied on each sub-area. Section 4 is concerned with explaining the method of extracting the data for energy and area coverage as the two decision criteria. Section 5 clarifies the general concepts of a MCDM method called Simple Additive Weighing (SAW) and then applies concepts of this method on the tiling-based path planning for the robot by means of defining a special fitness function. Finally, Section 6 is dedicated to a thorough analysis of the results in terms of fitness function values. In addition, a preference order for different theorems applied to different sub-areas is presented along with the best possible combination of tiling sets for the whole area. Section 7 presents an evaluation of the robot performance with and without the tiling. Consequently, Section 8 concludes the results and suggests possible works in the future.

\section{An Overview of the Experimental Environment}

An image of the experimental environment can be seen in Figure 1 which includes the hTetro robot and five obstacles. The outline of hTetro robot is shown in Figure 2, where all the four square-blocks are linked together with hinges. These blocks are driven by actuating the hinges with the smart servo motors mounted on it. The dimension of each hTetro block has a length, width, and height of $140 \mathrm{~mm}$, $140 \mathrm{~mm}$, and $55 \mathrm{~mm}$, respectively. Owing to the smaller and compact design structure of hTetro, each box is assigned to perform a specific function: Block 1 manages the locomotion of the robot, while control and power modules are located on block 2. Meanwhile, all the blocks are equipped with cleaning functions, but while the robot is performing reconfigurations, the cleaning function is turned off in order to devote the actuation energy fully to the servo motors controlling hinges. The aggregate weight of hTetro, including all its peripheral devices is approximately $3 \mathrm{~kg}$. For the mobility and transformation, hTetro is equipped with a set of six geared DC motors and three actuator servo motors, respectively. The three actuated servo motors, placed at hinges, are controlled to switch the robot between the seven possible configurations illustrated in Figure 3. For the actuation of hinge points during reconfiguration, Herkulex DRS-0101-7 V smart servos are utilized with a maximum rotation angle of $270^{\circ}$. The microcontroller used in hTetro is an Arduino Atmega 2560 16-Bit which performs three major functions:

- Receiving the command from the user by means of a smartphone which is connected wirelessly through a Bluetooth serial communication protocol. Arduino uses UART (Universal Asynchronous Receiver-Transmitter) for this communication. 
- Generating control signals to the motor driver which controls multiple DC motors; in addition, using PWM (Pulse Width Modulation), it controls the speed of the motor by providing the required current for the motor to run.

- Establishing full duplex serial communication with the servomotors where Arduino sends the control signals to the servo motors and simultaneously receives feedback from them.

For a thorough explanation of the mechanical design of hTetro and a detailed report on the content and functionality of each block, the reader is referred to [11,12]. The detailed system architecture of electrical conventions is given in Figure 4.

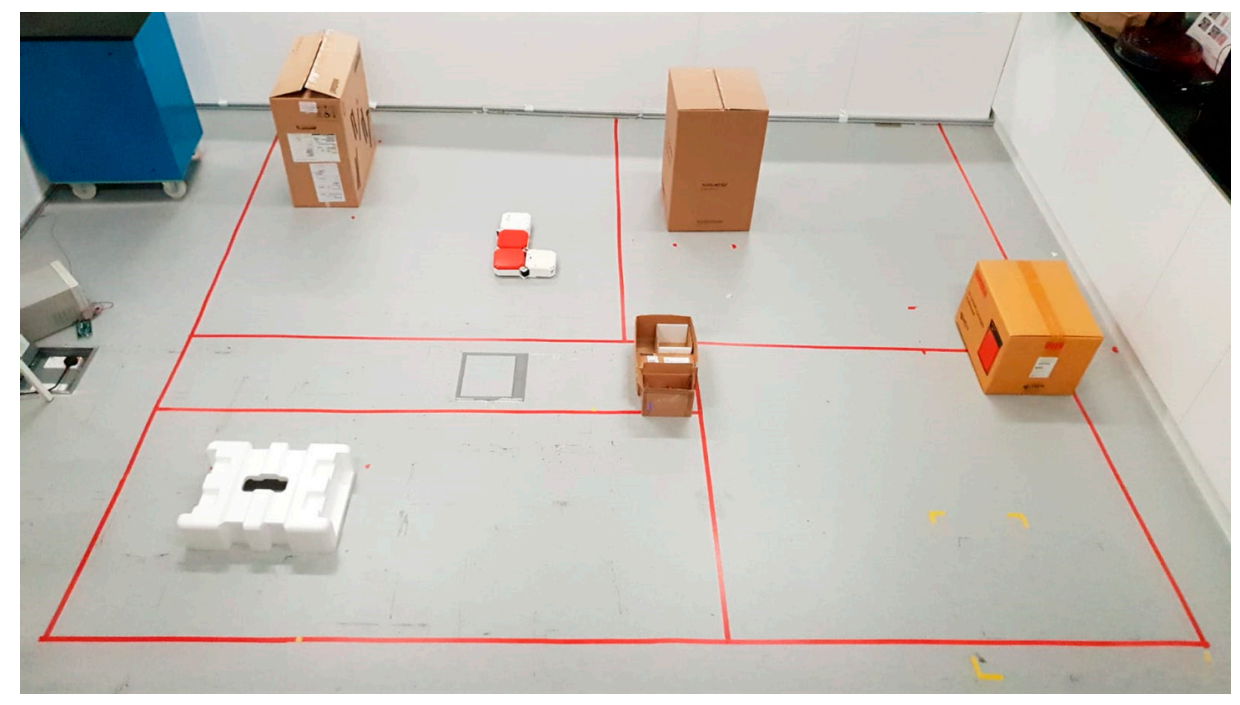

Figure 1. The experimental environment.

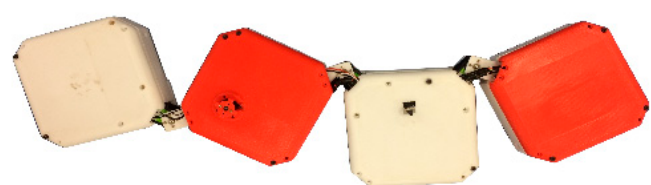

Figure 2. The hTetro architecture.

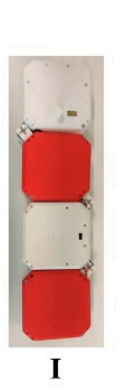

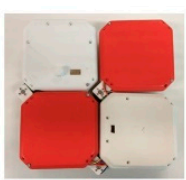

$\mathbf{0}$

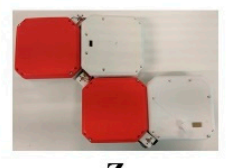

$\mathbf{Z}$

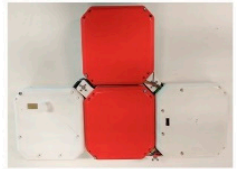

T

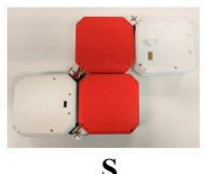

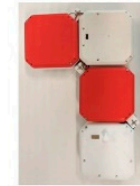

L

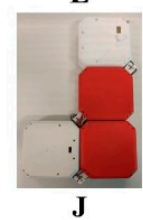

Figure 3. Seven one-sided tetrominoes. 


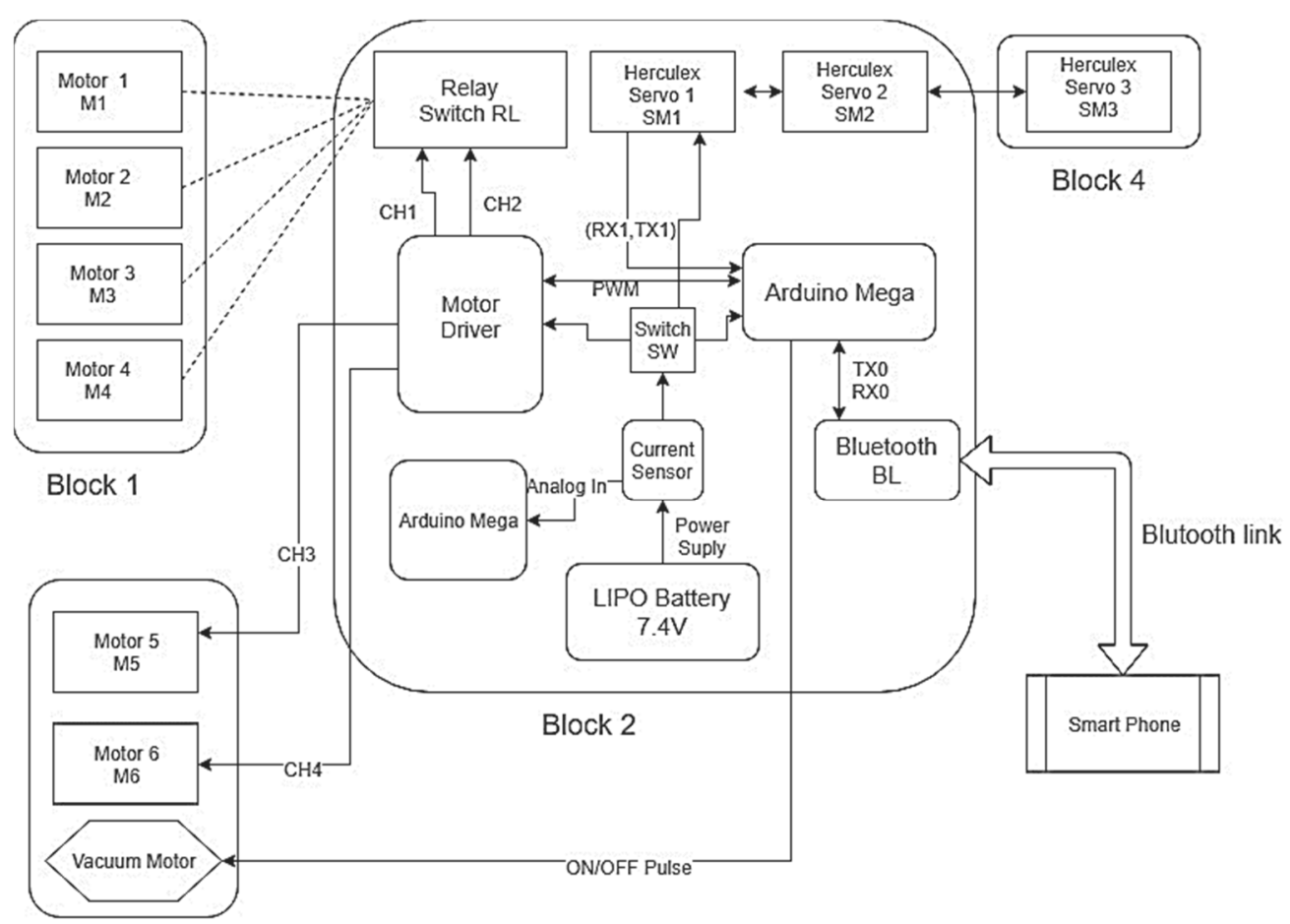

Block 3

Figure 4. Architecture of electrical conventions in hTetro.

\section{Polyomino Tiling Theory Applied to Our Robotic Platform}

Polyominoes are two-dimensional geometric figures that have equal sized constituent squares joined to each other by the edges [28]. They are also defined as n-ominoes, depending upon the number of squares that form the polyomino. The robotic platform used in this paper is hTetro that resembles a tetromino, i.e., a polyomino with four squares. As mentioned earlier, each of these hTetro square blocks moves about a hinge connection making it capable of reconfiguring into all seven one-sided shapes of a tetromino, as illustrated in Figure 3.

Polyomino tiling theory recommends mathematical theorems that allow for complete tiling with polyomino tiles without any overlap, via translations, rotations, and reflections, of tiles in the regions that fall within the constraints of the theorem. If these regions strictly adhere to the conditions of the theorems, tiling sets would not produce any gaps and should result in complete (exact) area coverage [28]. As with [13,14], in this paper, polyomino tiling theory is utilized to define the navigation strategies for reconfigurable floor-cleaning robots. To this end, initially, the entire space under cleaning is visualized as a large grid divided into congruent squares with dimensions that match the dimensions of robot blocks. These theorems can provide multiple tiling sets depending upon the dimensions of the region, obstacles contained, and the shapes permitted to be utilized in the theorem. Eventually, by reconfiguring from one shape to another, hTetro follows these tiling sets as a map for its navigation.

\subsection{Elaborating on the Applied Tiling Theorems}

The theorems enlisted below are applied to the experimental environment regarding the physical characteristics of hTetro cleaning robot. With the aim of applying the results to the experimental environment, only the concepts of the theorems are illustrated in this work. For further illustration on the proofs, the reader is referred to the references mentioned alongside each theorem statement.

In addition, before delving into theorem concepts, it should be mentioned that each theorem employs specific shapes of tetrominoes. Moreover, it should be clarified that, if the space is regarded as a grid divided into congruent square cells: 
- A modified rectangle is simply an $\mathrm{a} \times \mathrm{b}$ rectangle with both the upper-left and lower-right corner cells removed [29].

- A deficient rectangle means a rectangle in which some of its square cells are ignored or occupied by obstacles [30].

Theorem 1. A deficient square of area $3^{N} \times 3^{N}$, with one square removed, can be tiled by set $A$ [27] where Set $A$ includes tiles $O, T$, and $J$, as illustrated in Figure 3.

Theorem 2. A conventional rectangle $a \times b$ can be tiled by the set $B$ if, and only if, either of the following is true [29] where Set B includes tiles $Z, S$, and T, as illustrated in Figure 3.

1. One side is divisible by 4 ,

2. $a, b \equiv 2(\bmod 4)$ and $a+b>16$.

Theorem 3. A modified rectangle $a \times b$ can be tiled by the set $C$, if and only if, either of the following is true [29] where Set $C$ includes tiles $Z, S$, and T, as illustrated in Figure 3.

1. $a \equiv 2(\bmod 4)$ and $b$ is odd,

2. $b=2$ and $a \equiv 1(\bmod 4)$

Theorem 4. A deficient rectangle $a \times b$ can be tiled by set $D$, if and only if [30]:

1. $a$ and $b$ are odd and the rectangle is sized $(2 n+1) \times(2 n+1)$ when $n \geq 1$, and,

2. The missing cell in the deficient rectangle will have

- $\quad$ odd coordinates if the rectangle is sized $(4 m+1) \times(4 m+1)$ when $m \geq 1$, or

- even coordinates if the rectangle is sized $(4 m+3) \times(4 m+3)$ with $m \geq 1$.

where Set D includes $O, L$, and J tiles, as illustrated in Figure 3.

Furthermore, it should be mentioned that in order to reach a complete and exact tiling, the dimensions of the entire area must exactly follow the statement of the theorem and if they do not, a smaller rectangle inside the entire area could be considered that better fits the statement of the theorem. Also, in order to follow the requirements of the theorem, the entire area can be split into several smaller rectangles in a process called area decomposition.

\subsection{Area Decomposition Based on Furniture Layout}

As mentioned before, reconfigurability allows the robot to navigate around and under obstacles with different shapes. This results in accessing narrow spaces and inaccessible corners. However, this is possible while adhering to a tiling map as the navigation strategy; therefore, we can utilize the polyomino tiling theory. In order to apply these theorems perfectly for full area coverage, any space must satisfy the conditions of the theorem accurately, which is an unrealistic claim. That is why in this study, the entire area is manually decomposed into five sub-areas such that each sub-area will better satisfy the conditions of a theorem.

This decomposition depends on the position of each obstacle (furniture layout). In ideal circumstances, the robot must autonomously detect the presence, shape, and accessibility of the obstacles and tiny or unusual corners that require more attention. This experimental aspect is currently under investigation applying SLAM (Synchronous Localization And Mapping) and sensor fusion techniques. Consequently, depending upon the theorems in the robot's database, it will accordingly decompose the entire floor space into sub-areas for optimal area coverage. However, such decompositions do not always guarantee a perfect coverage of the sub-areas, resulting in untiled 
zones. That is why, optimizing the area decomposition based on furniture layout would serve as a future scope for this paper. Furthermore, the area decomposition will help to reduce the on-board computational cost working in each individual area with a restricted number of cells to be covered, which is the second reason supporting an area decomposition approach.

In this paper, the positions of randomly-placed obstacles are considered to manually divide the entire space into five sub-areas, as depicted in Figure 5. Notice that, in Figure 5, the sub-areas are numbered, and the yellow sections represent the obstacles. The floor space chosen for this paper is an area of $11.54 \mathrm{~m}^{2}$, such that the entire area can be covered exactly by 598 square cells. In correlation with the numbering on Figure 5, the respective areas of these distinct sub-areas are $2.058 \mathrm{~m}^{2}, 0.7056 \mathrm{~m}^{2}, 3.3124 \mathrm{~m}^{2}, 3.3124 \mathrm{~m}^{2}$, and $2.156 \mathrm{~m}^{2}$ without subtracting the area occupied by obstacles. After subtracting the obstacle-occupied area, the entire area to be cleaned is $10.5448 \mathrm{~m}^{2}$ which is the sum of $1.8228 \mathrm{~m}^{2}, 0.7056 \mathrm{~m}^{2}, 3.0184 \mathrm{~m}^{2}, 2.9988 \mathrm{~m}^{2}$, and $1.9992 \mathrm{~m}^{2}$ respectively for each numbered sub-area.

The general approach is to apply all four previously introduced theorems reasonably in each sub-area and propose consequent tiling sets that cover each sub-area. Finally, the resulting tiling sets will be analyzed in a Multi-Criteria Decision Making (MCDM) framework to test the effectiveness of these tiling theorems. Based on the MCDM results, the most efficient theorem for each sub-area is introduced that can result in a better balance between energy and area coverage.

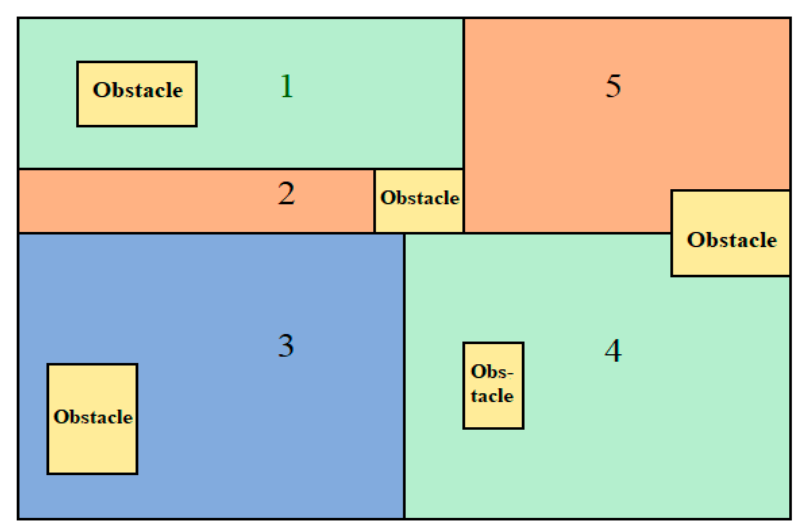

Figure 5. Area Decomposition based on furniture layout.

\subsection{The Resulting Tiling Sets (Navigation Strategies)}

Finally, all the aforementioned theorems are applied in each sub-area to generate five tiling sets which are indeed the robot's navigation strategies.

The proposed tiling sets are summarized in Figure 6, in which yellow rectangles represent obstacles and pink areas represent untiled zones. Each column of Figure 6 contains the results of applying a specific theorem on the whole area, and each row (under each column) represents a sample tiling solution (TS), applying the theorem mentioned in the header. Having four theorems with five tiling samples for each, we will have 20 tiling solutions for the whole area (100 tiling solutions to analyze for all sub-areas). In order to make it highly descriptive, the coloring of each sub-area in Figure 6 goes with Figure 5.

Due to the conditions imposed by each theorem, if the area in question does not perfectly match the conditions, the tiling set does not tile the entire area, resulting in distinct untiled zones within each sub-area. Realistically, the area covered by the tiled zones (tiled area) as shown in Figure 6 differs from the actual area spanned by the robot even when it is strictly following the tiling set for navigation. This is because, every time the robot reconfigures from one shape to another, some area may be traversed that is not tiled and does not exist in the tiling set (fake coverage), or some area will be traversed more than once during the reconfiguration process (re-coverage). However, as mentioned previously, the cleaning function of the robot is switched off while performing reconfigurations in 
order to devote the actuation energy fully to the servo motors controlling the hinges. This way, any unnecessary area that is not traversed for cleaning is disregarded.

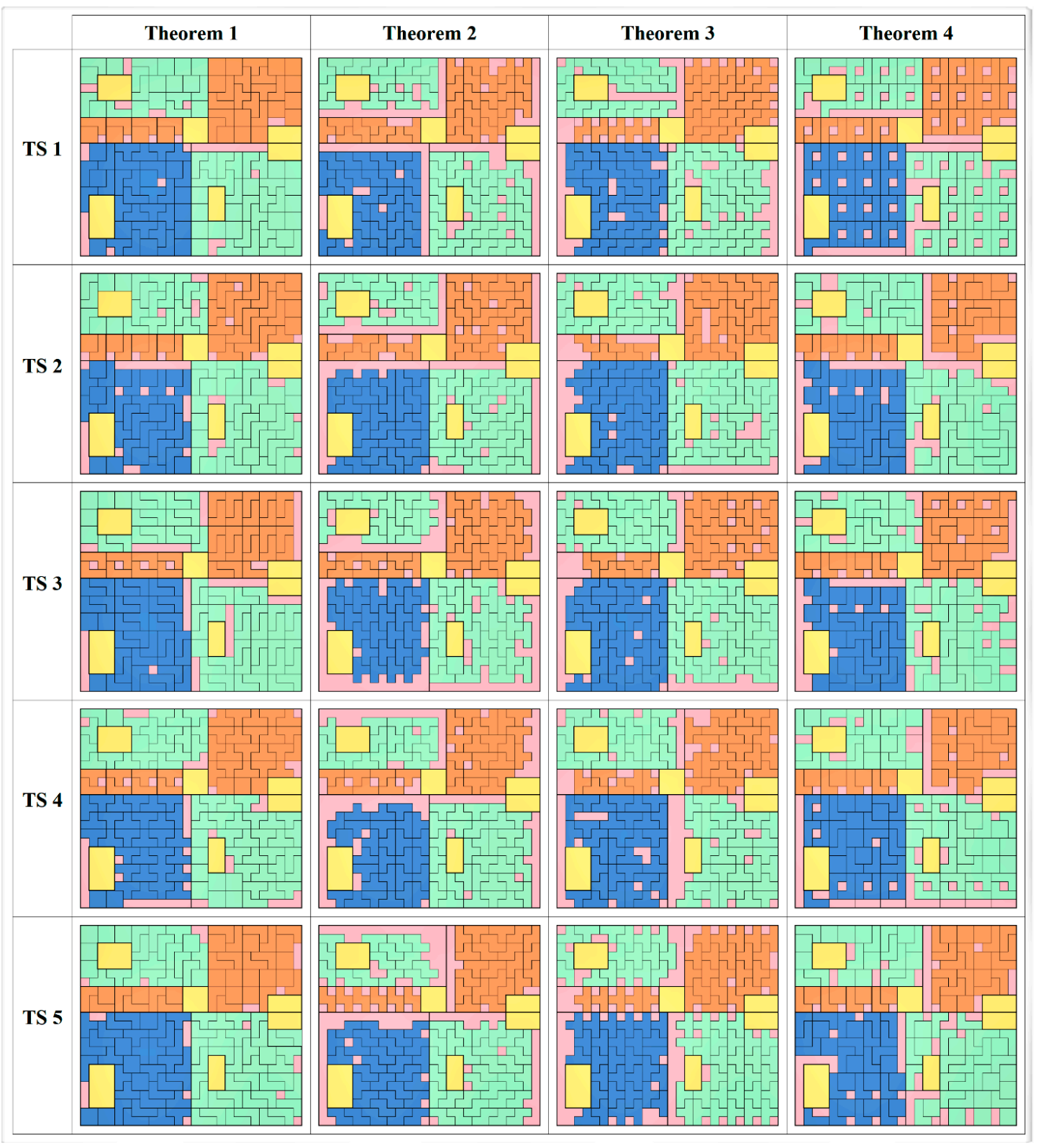

Figure 6. Five sets of tiling sets for all four theorems.

\section{Extracting the Data for Area Coverage and Energy}

The aim is to use the amounts of area coverage and energy consumption as decision criteria in a multi-criteria decision-making (MCDM) process.

On one hand, since all the blocks of hTetro are equipped with cleaning functions, tiling sets, as depicted in Figure 6, will represent area coverage values.

On the other hand, since the effect of time is not considered as a decision-criteria in this study, the consumed energy can be reported in terms of the consumed current [31,32]. Moreover, the consumed current by the robot can be estimated using a current estimation algorithm developed based on Newton-Raphson's algorithm $[33,34]$ which is illustrated by the pseudo code in Figure 7.

In each sub-area, the starting point of the reconfigurable robot is set as a fixed position for all the tiling patterns to avoid the difference in the starting voltage and current. When the robot begins to 
navigate, the energy values can be obtained with uniformity by fixing the common starting point and utilizing the same starting current for all the individual tiling sets [33].

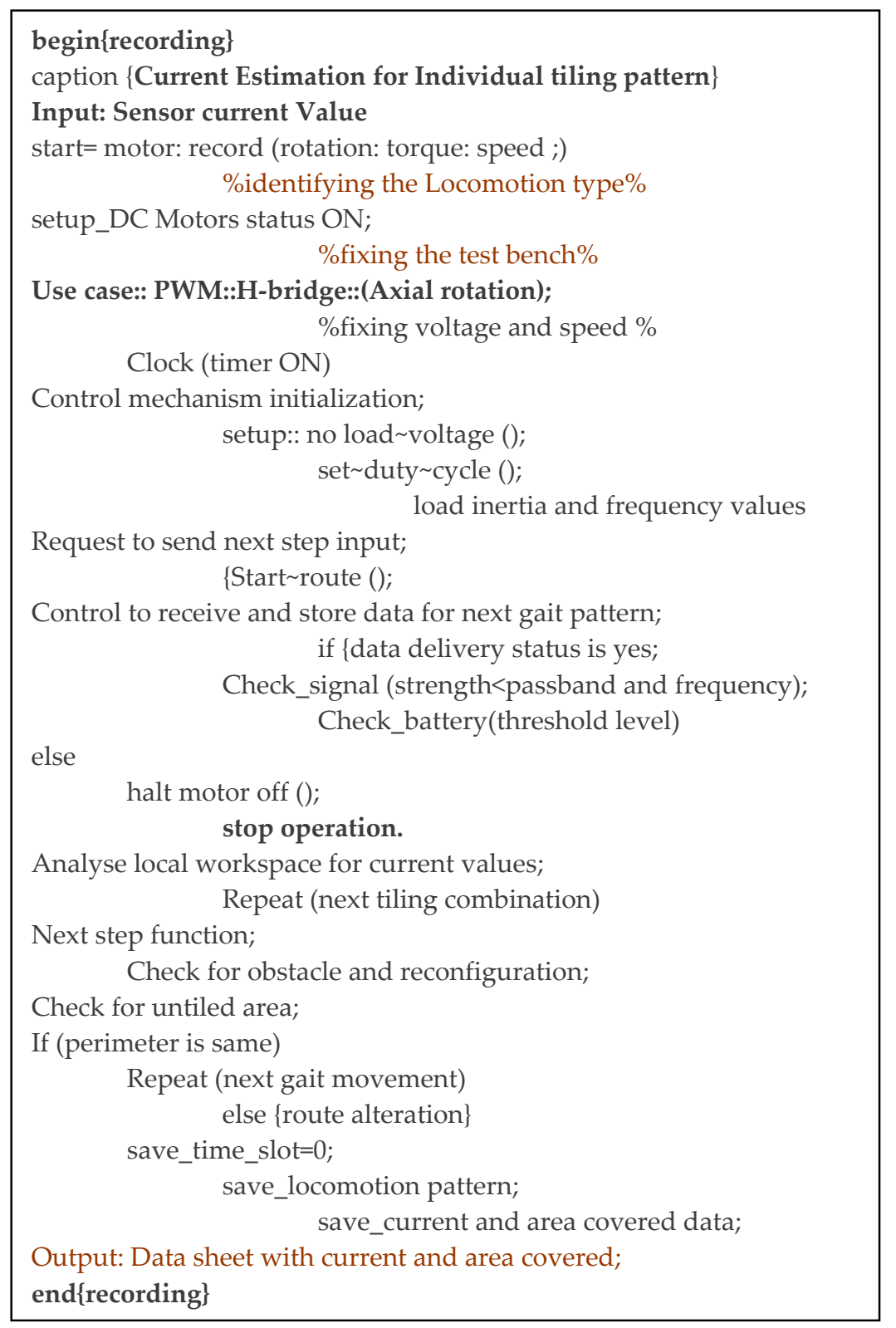

Figure 7. Pseudo code for the energy estimation scheme.

Using the pre-defined tiling set, given in Figure 6, the robot's energy consumption is calculated for individual gaits, while the amount of energy consumed by all the motors for a single-step movement containing all types of locomotion, shapeshifting, and angular movements, overall, is called the gait pattern [33]. Supported by the experimental results, and considering only gait patterns as input, the motor torque, no-load speed, current, and the rated DC voltages are obtained by allowing the robot to traverse within the given tiling set. In the experiments, the output voltage amplitude is taken as the same as the offset value of the DC motor and the duty cycle, while applying the PWM technique, is calculated for the full $12 \mathrm{~V}$ DC supply. The total current ratio is set as $7.4 \mathrm{~A}$ before starting the area coverage.

Generally, based on the Newton-Raphson algorithm, we developed an energy estimation scheme that characterizes the energy cost in terms of the current consumed with respect to specific locomotion 
gaits across all valid morphologies. This approach was adopted in our work to compute the current consumed by our hTetro robot for covering a given area.

Tables 1-5 summarize the area coverage and energy values (in both percentages and real values) for each sub-area applying different tiling sets given by the introduced theorems. In addition, it should be mentioned that the obs-free in the Tables 1-5 stand for the area which is the result of subtracting the area occupied by obstacles from the entire sub-area.

Table 1. Data for area coverage and energy for sub-area 1.

\begin{tabular}{|c|c|c|c|c|c|c|}
\hline & & & \multicolumn{2}{|c|}{ Area Coverage } & \multicolumn{2}{|c|}{ Energy } \\
\hline & & & Area \% & Real Value $\left(\mathrm{m}^{2}\right)$ & Energy \% & Real Value (A) \\
\hline \multirow{10}{*}{$\begin{array}{c}\text { Sub-Area } 1 \\
2.058 \mathrm{~m}^{2}\end{array}$} & \multirow{5}{*}{ Theorem 1} & Tiling set 1 & 90.322 & 1.6464 & 16.11 & 1.192 \\
\hline & & Tiling set 2 & 94.623 & 1.7248 & 15.09 & 1.117 \\
\hline & & Tiling set 3 & 86.021 & 1.568 & 14.90 & 1.103 \\
\hline & & Tiling set 4 & 90.322 & 1.6464 & 15.92 & 1.178 \\
\hline & & Tiling set 5 & 94.623 & 1.7248 & 15.01 & 1.111 \\
\hline & \multirow{5}{*}{ Theorem 2} & Tiling set 1 & 68.817 & 1.2544 & 15.60 & 1.419 \\
\hline & & Tiling set 2 & 68.817 & 1.2544 & 16.78 & 1.423 \\
\hline & & Tiling set 3 & 64.52 & 1.2548 & 14.31 & 1.431 \\
\hline & & Tiling set 4 & 68.817 & 1.2544 & 16.30 & 1.428 \\
\hline & & Tiling set 5 & 60.215 & 1.0926 & 15.76 & 1.428 \\
\hline \multirow{10}{*}{$\begin{array}{c}1.8228 \mathrm{~m}^{2} \\
\text { (obs-free) }\end{array}$} & \multirow{5}{*}{ Theorem 3} & Tiling set 1 & 77.419 & 1.4112 & 16.29 & 1.442 \\
\hline & & Tiling set 2 & 86.02 & 1.568 & 16.01 & 1.433 \\
\hline & & Tiling set 3 & 86.021 & 1.568 & 15.37 & 1.440 \\
\hline & & Tiling set 4 & 86.021 & 1.568 & 14.98 & 1.466 \\
\hline & & Tiling set 5 & 73.118 & 1.3328 & 15.24 & 1.441 \\
\hline & \multirow{5}{*}{ Theorem 4} & Tiling set 1 & 73.118 & 1.3328 & 15.75 & 1.449 \\
\hline & & Tiling set 2 & 88.172 & 1.6072 & 14.23 & 1.440 \\
\hline & & Tiling set 3 & 86.021 & 1.568 & 14.76 & 1.480 \\
\hline & & Tiling set 4 & 86.021 & 1.568 & 15.90 & 1.466 \\
\hline & & Tiling set 5 & 90.32 & 1.6464 & 15.12 & 1.473 \\
\hline
\end{tabular}

Table 2. Data for area coverage and energy for sub-area 2.

\begin{tabular}{|c|c|c|c|c|c|c|}
\hline & & & \multicolumn{2}{|c|}{ Area Coverage } & \multicolumn{2}{|c|}{ Energy } \\
\hline & & & Area $\%$ & Real Value $\left(\mathrm{m}^{2}\right)$ & Energy \% & Real Value (A) \\
\hline \multirow{10}{*}{$\begin{array}{c}\text { Sub-Area } 2 \\
0.7056 \mathrm{~m}^{2}\end{array}$} & \multirow{5}{*}{ Theorem 1} & Tiling set 1 & 88.888 & 0.6266 & 11.98 & 0.866 \\
\hline & & Tiling set 2 & 88.888 & 0.6266 & 12.55 & 0.929 \\
\hline & & Tiling set 3 & 88.888 & 0.6266 & 12.21 & 0.903 \\
\hline & & Tiling set 4 & 88.888 & 0.6266 & 12.02 & 0.889 \\
\hline & & Tiling set 5 & 100 & 0.7056 & 11.45 & 0.847 \\
\hline & \multirow{5}{*}{ Theorem 2} & Tiling set 1 & 88.888 & 0.6266 & 12.91 & 1.101 \\
\hline & & Tiling set 2 & 77.777 & 0.5488 & 12.11 & 1.126 \\
\hline & & Tiling set 3 & 88.888 & 0.6266 & 11.52 & 0.998 \\
\hline & & Tiling set 4 & 77.777 & 0.5488 & 12.10 & 1.012 \\
\hline & & Tiling set 5 & 66.666 & 0.4704 & 11.00 & 1.110 \\
\hline \multirow[t]{10}{*}{ No Obstacle } & \multirow{5}{*}{ Theorem 3} & Tiling set 1 & 55.555 & 0.3914 & 11.78 & 0.989 \\
\hline & & Tiling set 2 & 55.555 & 0.3914 & 12.41 & 0.961 \\
\hline & & Tiling set 3 & 66.666 & 0.4704 & 11.56 & 1.003 \\
\hline & & Tiling set 4 & 66.666 & 0.4704 & 12.30 & 1.120 \\
\hline & & Tiling set 5 & 55.555 & 0.3914 & 12.23 & 0.890 \\
\hline & \multirow{5}{*}{ Theorem 4} & Tiling set 1 & 88.888 & 0.6266 & 12.90 & 0.994 \\
\hline & & Tiling set 2 & 88.888 & 0.6266 & 11.89 & 1.102 \\
\hline & & Tiling set 3 & 88.888 & 0.6266 & 12.32 & 1.119 \\
\hline & & Tiling set 4 & 88.888 & 0.6266 & 12.12 & 1.103 \\
\hline & & Tiling set 5 & 88.888 & 0.6266 & 11.95 & 0.988 \\
\hline
\end{tabular}


Table 3. Data for area coverage and energy for sub-area 3.

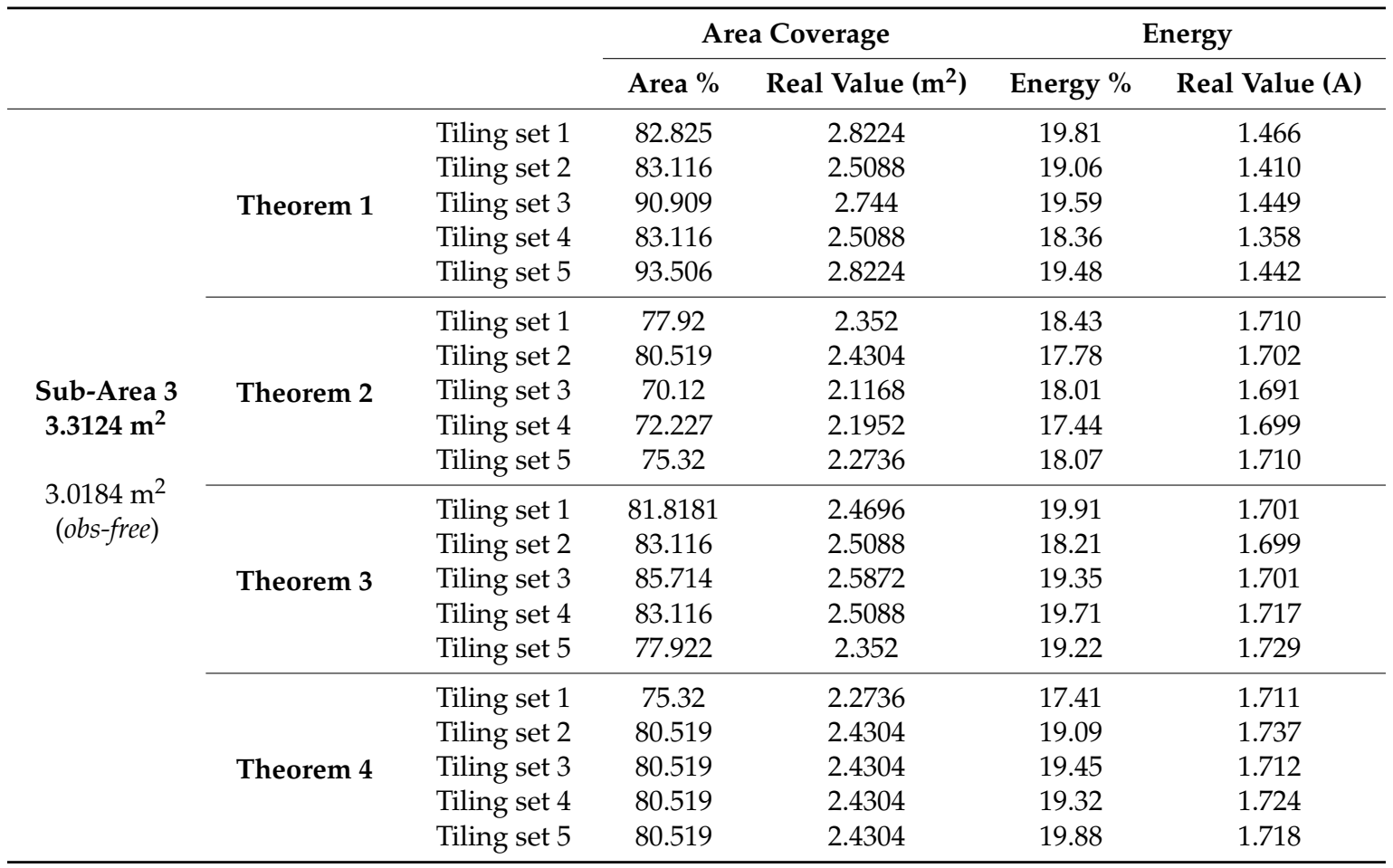

Table 4. Data for area coverage and energy for sub-area 4.

\begin{tabular}{|c|c|c|c|c|c|c|}
\hline & & & \multicolumn{2}{|c|}{ Area Coverage } & \multicolumn{2}{|c|}{ Energy } \\
\hline & & & Area \% & Real Value $\left(\mathrm{m}^{2}\right)$ & Energy \% & Real Value (A) \\
\hline \multirow{10}{*}{$\begin{array}{c}\text { Sub-Area } 4 \\
3.3124 \mathrm{~m}^{2}\end{array}$} & \multirow{5}{*}{ Theorem 1} & Tiling set 1 & 91.503 & 2.744 & 18.23 & 1.349 \\
\hline & & Tiling set 2 & 94.117 & 2.8224 & 16.79 & 1.242 \\
\hline & & Tiling set 3 & 86.2745 & 2.5872 & 17.10 & 1.265 \\
\hline & & Tiling set 4 & 91.503 & 2.744 & 17.39 & 1.287 \\
\hline & & Tiling set 5 & 96.732 & 2.9008 & 16.58 & 1.227 \\
\hline & \multirow{5}{*}{ Theorem 2} & Tiling set 1 & 77.124 & 2.3128 & 16.89 & 1.779 \\
\hline & & Tiling set 2 & 80.392 & 2.4108 & 17.66 & 1.767 \\
\hline & & Tiling set 3 & 73.202 & 2.1952 & 15.16 & 1.795 \\
\hline & & Tiling set 4 & 84.313 & 2.5284 & 16.78 & 1.801 \\
\hline & & Tiling set 5 & 81.045 & 2.4304 & 17.09 & 1.780 \\
\hline \multirow{10}{*}{$\begin{array}{c}2.9988 \mathrm{~m}^{2} \\
(\text { obs-free })\end{array}$} & \multirow{5}{*}{ Theorem 3} & Tiling set 1 & 81.04 & 2.4304 & 16.51 & 1.771 \\
\hline & & Tiling set 2 & 83.66 & 2.5088 & 16.66 & 1.770 \\
\hline & & Tiling set 3 & 86.274 & 2.5872 & 17.71 & 1.799 \\
\hline & & Tiling set 4 & 75.816 & 2.2736 & 15.39 & 1.787 \\
\hline & & Tiling set 5 & 78.431 & 2.352 & 17.41 & 1.770 \\
\hline & \multirow{5}{*}{ Theorem 4} & Tiling set 1 & 73.202 & 2.1952 & 15.89 & 1.799 \\
\hline & & Tiling set 2 & 83.66 & 2.5088 & 17.55 & 1.769 \\
\hline & & Tiling set 3 & 83.66 & 2.5088 & 16.70 & 1.767 \\
\hline & & Tiling set 4 & 81.045 & 2.4304 & 17.45 & 1.775 \\
\hline & & Tiling set 5 & 83.66 & 2.5088 & 16.10 & 1.778 \\
\hline
\end{tabular}


Table 5. Data for area coverage and energy for sub-area 5.

\begin{tabular}{|c|c|c|c|c|c|c|}
\hline & & & \multicolumn{2}{|c|}{ Area Coverage } & \multicolumn{2}{|c|}{ Energy } \\
\hline & & & Area \% & Real Value $\left(\mathrm{m}^{2}\right)$ & Energy \% & Real Value (A) \\
\hline \multirow{10}{*}{$\begin{array}{c}\text { Sub-Area } 5 \\
2.156 \mathrm{~m}^{2}\end{array}$} & \multirow{5}{*}{ Theorem 1} & Tiling set 1 & 98.039 & 1.96 & 18.30 & 1.354 \\
\hline & & Tiling set 2 & 94.117 & 1.8816 & 18.68 & 1.382 \\
\hline & & Tiling set 3 & 90.196 & 1.8032 & 19.46 & 1.440 \\
\hline & & Tiling set 4 & 94.117 & 1.8816 & 20.04 & 1.483 \\
\hline & & Tiling set 5 & 94.117 & 1.8816 & 19.23 & 1.4237 \\
\hline & \multirow{5}{*}{ Theorem 2} & Tiling set 1 & 81.372 & 1.6268 & 19.09 & 1.489 \\
\hline & & Tiling set 2 & 86.27 & 1.7248 & 20.31 & 1.491 \\
\hline & & Tiling set 3 & 82.352 & 1.6364 & 20.80 & 1.511 \\
\hline & & Tiling set 4 & 82.352 & 1.6464 & 18.31 & 1.534 \\
\hline & & Tiling set 5 & 86.28 & 1.7248 & 21.03 & 1.487 \\
\hline \multirow{10}{*}{$\begin{array}{c}1.9992 \mathrm{~m}^{2} \\
(\text { obs-free })\end{array}$} & \multirow{5}{*}{ Theorem 3} & Tiling set 1 & 90.19 & 1.8032 & 20.53 & 1.512 \\
\hline & & Tiling set 2 & 94.117 & 1.8816 & 19.41 & 1.490 \\
\hline & & Tiling set 3 & 90.196 & 1.8032 & 19.05 & 1.506 \\
\hline & & Tiling set 4 & 86.27 & 1.7248 & 19.69 & 1.493 \\
\hline & & Tiling set 5 & 86.28 & 1.7248 & 21.10 & 1.510 \\
\hline & \multirow{5}{*}{ Theorem 4} & Tiling set 1 & 78.43 & 1.568 & 17.26 & 1.497 \\
\hline & & Tiling set 2 & 78.43 & 1.568 & 17.87 & 1.487 \\
\hline & & Tiling set 3 & 86.274 & 1.7248 & 20.33 & 1.503 \\
\hline & & Tiling set 4 & 78.431 & 1.568 & 17.65 & 1.480 \\
\hline & & Tiling set 5 & 82.352 & 1.6464 & 19.77 & 1.501 \\
\hline
\end{tabular}

\section{Multi-Criteria Decision Making}

\subsection{Simple Additive Weighing (SAW) Method-A Very Brief Review}

Owing to its simplicity, the SAW method is probably the most popular and most commonly used method for multiple-criteria decision making (MCDM), especially in single-dimensional problems. If there are $\mathrm{M}$ alternatives and $\mathrm{N}$ criteria then, the best alternative is the one that satisfies Equation (1).

$$
A^{*}=\left\{A_{i} \mid \max _{i} \sum_{j=1}^{N} w_{j} r_{i j}\right\} i=1,2, \ldots, M
$$

where, $N$ is the number of decision criteria, $r_{i j}$ is the normalized value of the $i$-th alternative in terms of the $j$-th criterion, and $w_{j}$ is the weight of importance of the $j$-th criterion. The values are normalized in order to make it possible to add the measurements with different units.

\subsection{SAW Concepts Applied to Tiling-Based Path Planning}

The energy consumption level is a key factor of any electromechanical system, and for cleaning applications, the amount of area coverage signifies another aspect of the performance. Here, we want to apply MCDM regarding these key factors (area and energy) as the decision-making criteria. As mentioned above, an area decomposition is defined based on the obstacle layout and the whole environment is divided into five sub-areas, as shown in Figure 5. Then, the four introduced tiling theorems are applied to each sub-area and five tiling sets are devised for each theorem in each sub-area. Regarding the given tiling sets for each sub-area, the results for the decision criteria are summarized within Tables 1-5. The final aim is to introduce a superior theorem for each sub-area; the theorem which could best tile the area in terms of area coverage and energy consumption. In order to do this, a fitness function for each given tiling set is described, as in Equation (2), in which $c_{1}$ is a positive weighting factor and $c_{2}$ is a negative weighting factor since we would like to increase the area coverage and decrease the energy utilization and create a balance between the two. For now, we assume that the area coverage and energy are of the same importance for the user and assume the importance 
weights $c_{1}$ and $c_{2}$ are equally 0.5 . In order to make the concepts of area and energy commensurable and make the addition possible, first we must normalize the values or we can simply use the values in percentage, which is representative of the real value regardless of units.

$$
f(s)=c_{1} A(s)-c_{2} E(s)
$$

\section{Result Analysis and Discussion}

In order to apply the proposed approach, Tables 1-5 are considered as the input data. For each sub-area, we have 20 given tiling sets, i.e., 20 alternatives: Four theorems that each give five tiling sets. In each sub-area, the value of the fitness function for each of the alternatives is calculated through (2), and consequently, making a comparison amongst the fitness values gives the best tiling set for each sub-area. In addition, not only a preference order between the individual tiling sets is suggested, but also a theorem that can better handle the environment is introduced based on sum of rankings for the tiling sets. Tables 6-10 summarize the ranking of the tiling sets based on the fitness function.

Consequently, based on the sum of rankings summarized in Tables 6-10, the preference order of the Theorems for each sub-area is given by the following (the option with the least sum of rankings appears to be the best).

- $\quad$ For sub-area 1: Theorem $1>$ Theorem $4>$ Theorem $3>$ Theorem 2

- $\quad$ For sub-area 2: Theorem $1>$ Theorem $4>$ Theorem $2>$ Theorem 3

- $\quad$ For sub-area 3: Theorem $1>$ Theorem $3>$ Theorem $4>$ Theorem 2

- $\quad$ For sub-area 4: Theorem $1>$ Theorem $3>$ Theorem $4>$ Theorem 2

- For sub-area 5: Theorem $1>$ Theorem $3>$ Theorem $2>$ Theorem 4

Table 6. Ranking of the tiling sets based on the fitness value for sub-area 1.

\begin{tabular}{cccc}
\hline Sub-Area 1 & & Rank & Rank Sum \\
\hline \multirow{4}{*}{ Theorem 1 } & Tiling set 1 & 5 & \\
& Tiling set 2 & 2 & 20 \\
& Tiling set 3 & 8 & \\
& Tiling set 4 & 4 & \\
& Tiling set 5 & 1 & \\
Theorem 2 & Tiling set 1 & 16 & \\
& Tiling set 2 & 18 & \\
& Tiling set 3 & 19 & \\
& Tiling set 4 & 17 & \\
& Tiling set 5 & 20 & \\
Theorem 3 & Tiling set 1 & 13 & \\
& Tiling set 2 & 12 & \\
& Tiling set 3 & 10 & \\
& Tiling set 4 & 9 & \\
& Tiling set 5 & 14 & \\
\hline \multirow{5}{*}{ Theorem 4 } & Tiling set 1 & 15 & \\
& Tiling set 2 & 6 & \\
& Tiling set 3 & 7 & \\
& Tiling set 4 & 11 & \\
& Tiling set 5 & 3 & \\
\hline
\end{tabular}


Table 7. Ranking of the tiling sets based on the fitness value for sub-area 2.

\begin{tabular}{cccc}
\hline Sub-Area 2 & & Rank & Rank Sum \\
\hline \multirow{4}{*}{ Theorem 1 } & Tiling set 1 & 5 & \\
& Tiling set 2 & 10 & 30 \\
& Tiling set 3 & 8 & \\
& Tiling set 4 & 6 & \\
& Tiling set 5 & 1 & \\
Theorem 2 & Tiling set 1 & 12 & \\
& Tiling set 2 & 14 & \\
& Tiling set 3 & 2 & \\
& Tiling set 4 & 13 & \\
& Tiling set 5 & 15 & \\
Theorem 3 & Tiling set 1 & 18 & \\
& Tiling set 2 & 20 & \\
& Tiling set 3 & 16 & \\
& Tiling set 4 & 17 & \\
& Tiling set 5 & 19 & \\
\hline \multirow{5}{*}{ Theorem 4 } & Tiling set 1 & 11 & \\
& Tiling set 2 & 3 & \\
& Tiling set 3 & 9 & \\
& Tiling set 4 & 7 & \\
\hline
\end{tabular}

Table 8. Ranking of the tiling sets based on the fitness value for sub-area 3.

\begin{tabular}{cccc}
\hline Sub-Area 3 & & Rank & Rank Sum \\
\hline \multirow{4}{*}{ Theorem 1 } & Tiling set 1 & 8 & \\
& Tiling set 2 & 6 & 22 \\
& Tiling set 3 & 2 & \\
& Tiling set 4 & 5 & \\
& Tiling set 5 & 1 & \\
Theorem 2 & Tiling set 1 & 15 & \\
& Tiling set 2 & 9 & \\
& Tiling set 3 & 20 & \\
& Tiling set 4 & 19 & \\
& Tiling set 5 & 18 & \\
Theorem 3 & Tiling set 1 & 10 & \\
& Tiling set 2 & 4 & \\
& Tiling set 3 & 3 & \\
& Tiling set 4 & 7 & \\
& Tiling set 5 & 16 & \\
\hline \multirow{2}{*}{ Theorem 4 } & Tiling set 1 & 17 & \\
& Tiling set 2 & 11 & \\
& Tiling set 3 & 13 & \\
& Tiling set 4 & 12 & \\
\hline
\end{tabular}


Table 9. Ranking of the tiling sets based on the fitness value for sub-area 4 .

\begin{tabular}{cccc}
\hline Sub-Area 4 & & Rank & Rank Sum \\
\hline \multirow{3}{*}{ Theorem 1 } & Tiling set 1 & 4 & \\
& Tiling set 2 & 2 & 15 \\
& Tiling set 3 & 5 & \\
& Tiling set 4 & 3 & \\
& Tiling set 5 & 1 & \\
Theorem 2 & Tiling set 1 & 18 & \\
& Tiling set 2 & 15 & \\
& Tiling set 3 & 19 & \\
& Tiling set 4 & 8 & \\
& Tiling set 5 & 13 & \\
Theorem 3 & Tiling set 1 & 12 & \\
& Tiling set 2 & 9 & \\
& Tiling set 3 & 6 & \\
& Tiling set 4 & 17 & \\
& Tiling set 5 & 16 & \\
\hline \multirow{3}{*}{ Theorem 4 } & Tiling set 1 & 20 & \\
& Tiling set 2 & 11 & \\
& Tiling set 3 & 10 & \\
& Tiling set 4 & 14 & \\
\hline
\end{tabular}

Table 10. Ranking of the tiling sets based on the fitness value for sub-area 5 .

\begin{tabular}{cccc}
\hline Sub-Area 5 & & Rank & Rank Sum \\
\hline \multirow{4}{*}{ Theorem 1 } & Tiling set 1 & 1 & \\
& Tiling set 2 & 2 & 18 \\
& Tiling set 3 & 7 & \\
& Tiling set 4 & 5 & \\
& Tiling set 5 & 3 & \\
Theorem 2 & Tiling set 1 & 16 & \\
& Tiling set 2 & 10 & \\
& Tiling set 3 & 17 & \\
& Tiling set 4 & 14 & \\
& Tiling set 5 & 12 & \\
Theorem 3 & Tiling set 1 & 8 & \\
& Tiling set 2 & 4 & \\
& Tiling set 3 & 6 & \\
& Tiling set 4 & 9 & \\
& Tiling set 5 & 13 & \\
\hline \multirow{3}{*}{ Theorem 4 } & Tiling set 1 & 18 & \\
& Tiling set 2 & 20 & \\
& Tiling set 3 & 11 & \\
& Tiling set 4 & 19 & \\
\hline
\end{tabular}

On the other hand, a graphical analysis of the results is illustrated in Figures 8-12 in which the different tiling sets given by different tiling theorems are depicted based on the ranking of their corresponding fitness function. In the given bar graphs, for each sub-area, the results given by Theorem 1, Theorem 2, Theorem 3, and Theorem 4 are shown in red, green, blue and yellow bars, respectively. Meanwhile, regarding the vertical axis, there is a labeling applied to the real fitness values to increase the visibility for comparison: The first ranking tiling set (with highest fitness function) is given the value 20 and the last ranking one (with lowest fitness function) is given the value of 1 . Moreover, in the horizontal axis, the expressions Th i-Ts j stands for Theorem i-Tiling set j. It is clear that Figures 8-12 
introduce a precise preference order between each given tiling set, regardless of the theorem applied. However, the overall performance of each theorem is visualized through the total area occupied by the corresponding colored bars.

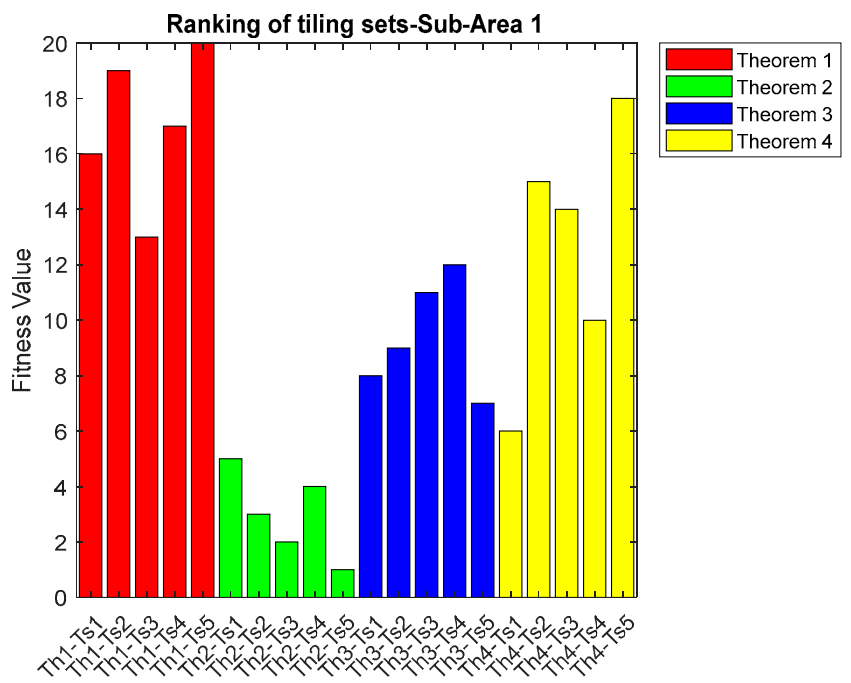

Figure 8. Ranking of fitness values for tiling sets under each theorem-sub-area 1.

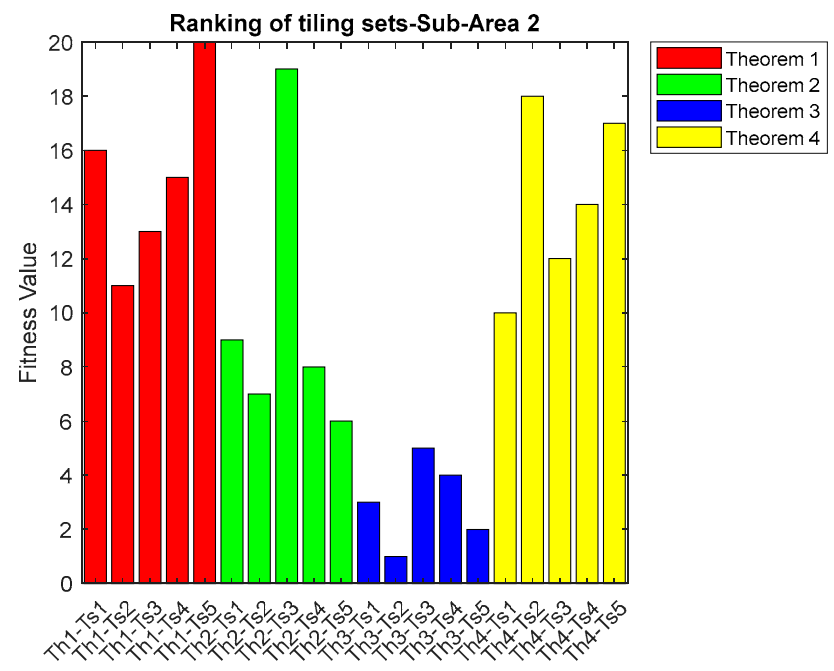

Figure 9. Ranking of fitness values for tiling sets under each theorem-sub-area 2.

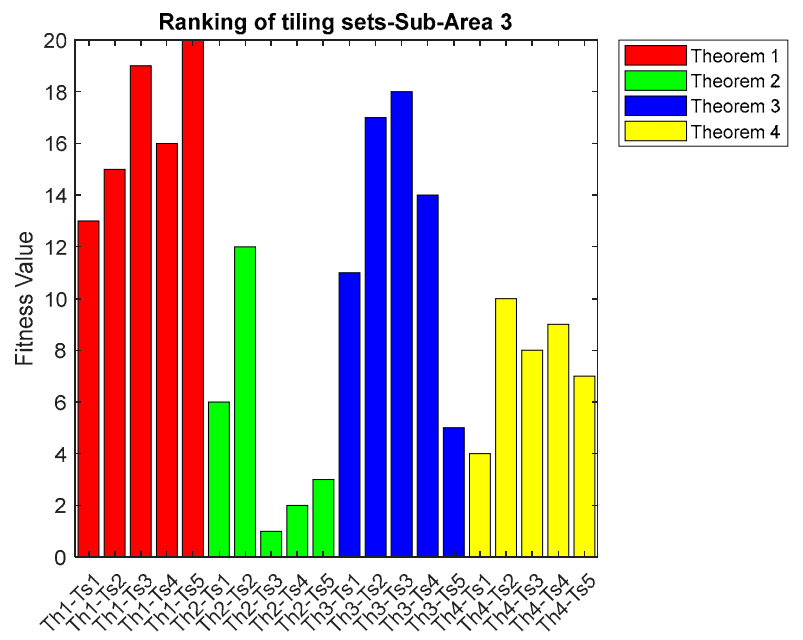

Figure 10. Ranking of fitness values for tiling sets under each theorem-sub-area 3. 


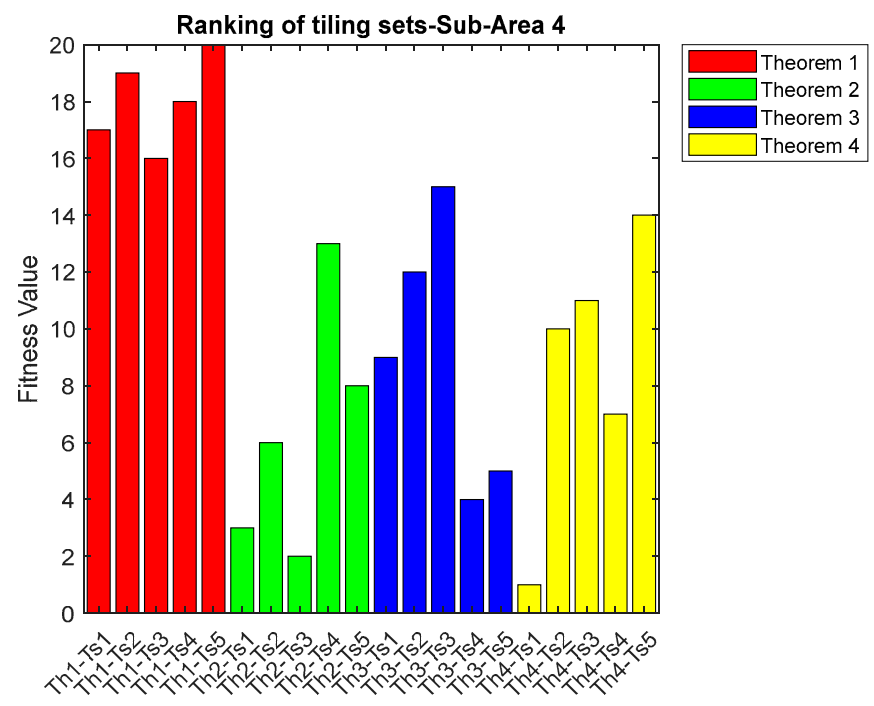

Figure 11. Ranking of fitness values for tiling sets under each theorem-sub-area 4.

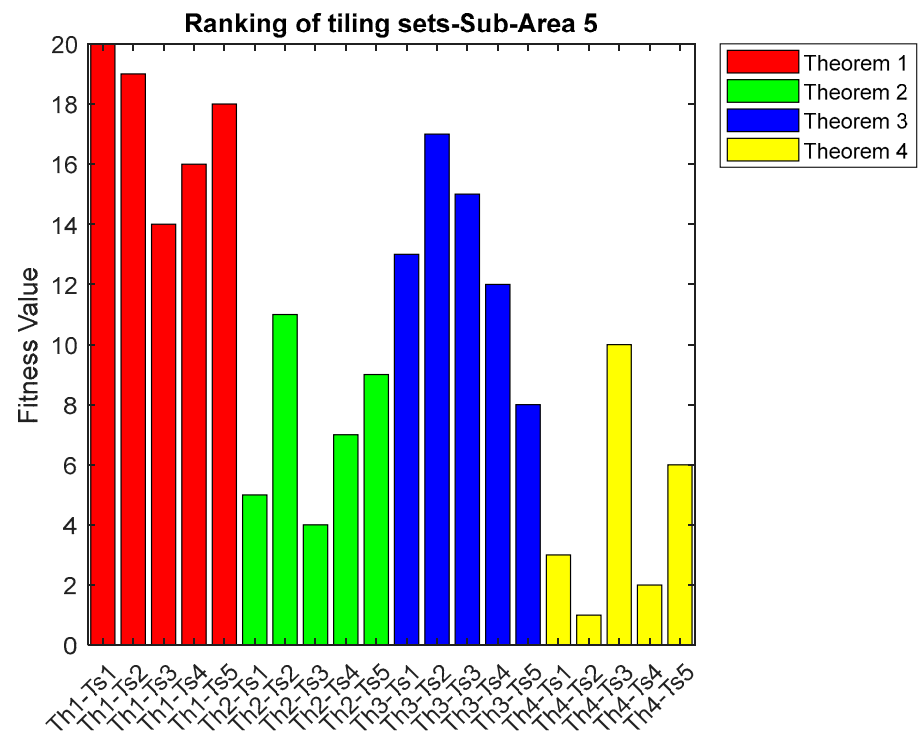

Figure 12. Ranking of fitness values for tiling sets under each theorem-sub-area 5.

It is evident that in all five sub-areas, Theorem 1 has been performing better than other theorems with regard to fulfilling the balance between area coverage and energy consumption. To add a further illustration, a sketch of the best given tiling set is shown in Figure 13, which shows the following tiling sets together:

- $\quad$ Tiling set 5 given by Theorem 1 for sub-area 1 ,

- $\quad$ Tiling set 5 given by Theorem 1 for sub-area 2 ,

- $\quad$ Tiling set 5 given by Theorem 1 for sub-area 3,

- $\quad$ Tiling set 5 given by Theorem 1 for sub-area 4 ,

- $\quad$ Tiling set 1 given by Theorem 1 for sub-area 5 ,

With reference to Tables $1-5$, the whole additive energy value for this composition of the highest-ranking tiling sets is $5.981 \mathrm{~A}$ and the whole area covered by this superior tiling set is $10.1136 \mathrm{~m}^{2}$ (95.9\% of the entire area required to be cleaned). As it can be seen, the decision goal is fulfilled to a satisfying extent in terms of the defined criteria since the entire energy can be provided by a one-time battery charge (less than $7.4 \mathrm{~A}$ ), and the area coverage is the highest available one. 

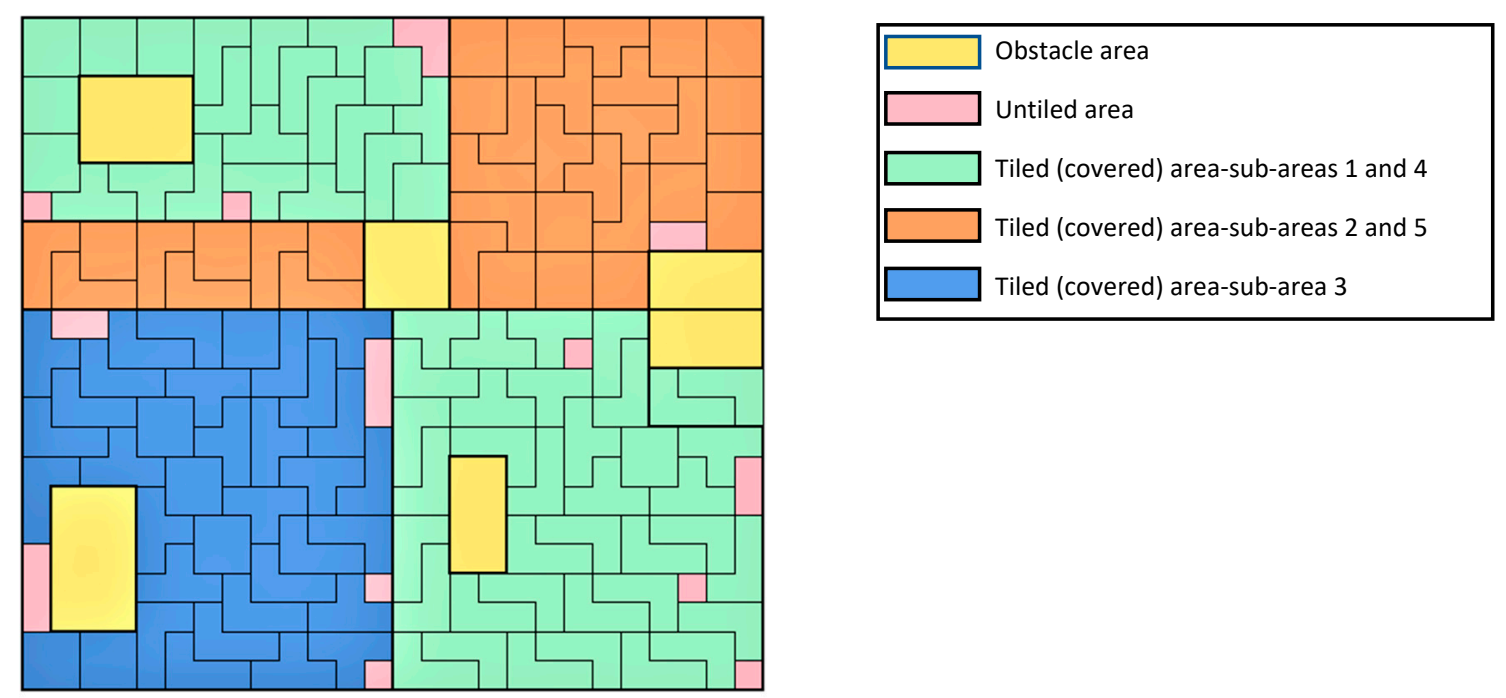

Figure 13. A composition of best tiling sets for each sub-area.

\section{Evaluating the Robot Performance with and without the Tiling}

Providing access to narrow and difficult spaces, self-reconfiguration is totally reasonable for maximizing the area coverage. However, shape-shifting may sound a waste of energy. In this section, a comparison is illustrated with a fixed-morphology robot with the same size ( $\mathrm{O}$ reconfiguration in Figure 3) that follows no tiling theory but tries to cover the area like the current commercialized cleaning robots. Figure 14 shows nine such sweeping solutions for the entire experimental environment in which each of the solutions appears with the corresponding numbering and each yellow square is an $\mathrm{O}$ morphology, as depicted in Figure 3. In addition, the gray area is the area totally inaccessible and the purple area is the area that is accessible at the expense of re-coverage. The total area coverage percentage and utilized energy amount in each sweeping solution is summarized in Table 11 in which the reported area is the covered area with avoidance of re-coverage, i.e., the whole area subtracted by the gray and purple areas and the area occupied by obstacles.
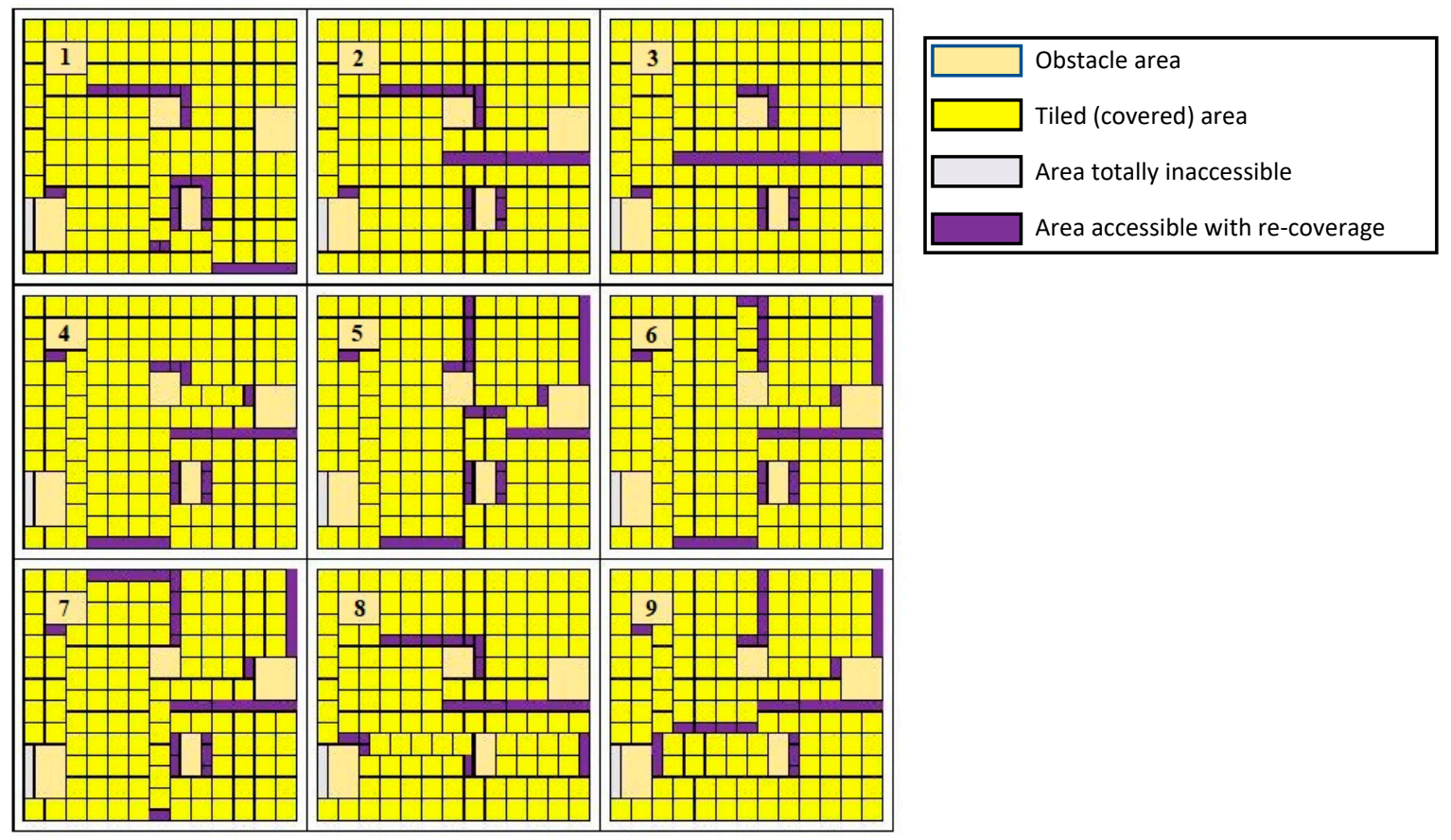

Figure 14. Sample sweeping solutions with fixed morphology and no tiling theory. 
Table 11. Energy and area coverage for fixed-morphologies with no tiling theorem.

\begin{tabular}{ccc}
\hline Sweeping Solution & Area-Coverage (\%) & Energy (A) \\
\hline $\mathbf{1}$ & $496 / 538=92.19 \%$ & 5.23 \\
$\mathbf{2}$ & $496 / 538=92.19 \%$ & 5.44 \\
$\mathbf{3}$ & $496 / 538=92.19 \%$ & 5.87 \\
$\mathbf{4}$ & $496 / 538=92.19 \%$ & 5.91 \\
$\mathbf{5}$ & $484 / 538=89.96 \%$ & 6.67 \\
$\mathbf{6}$ & $484 / 538=89.96 \%$ & 6.312 \\
$\mathbf{7}$ & $484 / 538=89.96 \%$ & 6.18 \\
$\mathbf{8}$ & $496 / 538=92.19 \%$ & 6.22 \\
$\mathbf{9}$ & $484 / 538=89.96 \%$ & 6.48 \\
\hline
\end{tabular}

Considering Table 11, it is obvious that the fixed-morphology performance was not as energy-efficient as expected and its energy consumption for sweeping the entire space is almost the same as the best alternative selected by MCDM (5.981 A) or even higher (worse) than that in some cases (sweeping solution 5, 6, 7, 8, 9). However, the shape-shifting morphology evidently results in higher area coverage. The results in Table 11 also justify this fact: In a fixed-morphology scheme with no tiling-path-planning, we have, at most, $92 \%$ area coverage, but the approach presented in this paper results in almost $96 \%$ area coverage, see Figure 13.

To summarize, when a higher area coverage is guaranteed by self-reconfiguration, and the energy consumption in a fixed-morphology scheme is at the level of shape-shifting morphology (slightly better: $5.98-5.23=0.75$ (A) or even worse: $5.98-6.67=-0.7(\mathrm{~A})$ ), it sounds reasonable to apply the MCDM-supported tiling path-planning even though it requires a slight compromise in energy consumption.

\section{Conclusions}

By providing self-reconfiguration abilities for the cleaning robot, it can cope with limitations associated with sweeping narrow corridors and far-to-access corners, making self-reconfigurable robots very interesting for use as cleaners. In this regard, a Tetris-inspired mechanism has already been proposed in our previous studies and, additionally, polyomino tiling theory has been introduced as a means of path planning for such a mechanism. Previous studies in this field, have only considered area coverage as the performance metric for the cleaning robot. Moreover, besides the amount of cleaned area, the amount of battery usage (energy) is a significant issue. In this paper, we applied a MCDM approach aimed at efficient cleaning in terms of energy and area coverage. This study has moved a step forward regarding the quest to find the best alternative among given tiling maps in order to orient the robot towards the optimal navigation.

To this end, in this study, four tiling set theorems are utilized to define the navigation maps for the robot. Before applying the tiling theorems, the entire area is decomposed to five sub-areas based on furniture layout. Then, the tiling theorems are applied to each sub-area separately to generate several distinguished tiling sets. These are the alternatives analyzed from the area and energy viewpoint. To balance the facing trade-off, a MCDM approach is applied by defining a fitness function in terms of the decision criteria and finally, all the alternatives are ranked in terms of the resulting fitness function. Based on the results of this ranking, one of the most recent theorems stood out, showing a more efficient performance throughout all the sub-areas. Furthermore, a comparison between the performance of a fixed-morphology and a shape-shifting mechanism is presented that further suggests the MCDM-supported tiling path planning.

As a scope of future work, the area decomposition method can be improved based on cellular decomposition supported by SLAM. Additionally, here, only the effect of area coverage and energy are investigated as the decision criteria, while time is also an influencing factor in the hectic timetables of people nowadays. Considering the required time as another aspect of the efficiency of a cleaning application could be another aspect of future work. On the other hand, in this study, both the decisive 
factors in a MCDM process are regarded to be of the same value to the user. Defining the partial importance of the decision criteria based on user preferences is actually not difficult to imagine. This can also open an opportunity for incorporating fuzzy logic.

Author Contributions: Conceptualization, M.K.; Formal analysis, M.K.; Investigation, M.K. and M.A.P.; Methodology, M.K., M.R.E. and M.A.P.; Project administration, M.K. and M.R.E.; Resources, M.R.E.; Software, M.K. and M.A.; Supervision, M.K. and M.R.E.; Writing—original draft, M.K., M.R.E., M.A.P., M.A. and V.P.

Acknowledgments: This research was funded by the National Robotics R \& D Program Office, Singapore, under the Grant No. RGAST1702, Singapore University of Technology and Design (SUTD) which are gratefully acknowledged to conduct this research work.

Conflicts of Interest: The authors declare no conflict of interest.

\section{References}

1. Vaussard, F.; Fink, J.; Bauwens, V.; Rétornaz, P.; Hamel, D.; Dillenbourg, P.; Mondada, F. Lessons learned from robotic vacuum cleaners entering the home ecosystem. Robot. Autom. Syst. 2014, 62, 376-391. [CrossRef]

2. Palleja, T.; Tresanchez, M.; Teixido, M.; Palacin, J. Modeling floor-cleaning coverage performances of some domestic mobile robots in a reduced scenario. Robot. Autom. Syst. 2010, 58, 37-45. [CrossRef]

3. Gao, X.; Li, K.; Wang, Y.; Men, G.; Zhou, D.; Kikuchi, K. A floor cleaning robot using Swedish wheels. In Proceedings of the IEEE International Conference on Robotics and Biomimetics (ROBIO), Sanya, China, 15-18 December 2007.

4. Yang, S.X.; Luo, C. A neural network approach to complete coverage path planning. IEEE Trans. Syst. Man Cybern. Part B 2004, 34, 718-724. [CrossRef]

5. Wong, S.C.; Middleton, L.; MacDonald, B.A.; Auckland, N. Performance metrics for robot coverage tasks. In Proceedings of the Australasian Conference on Robotics and Automatio, Auckland, New Zealand, 27-29 November 2002.

6. Ahmadi, M.; Stone, P. A multi-robot system for continuous area sweeping tasks. In Proceedings of the IEEE International Conference on Robotics and Automation (ICRA), Orlando, FI, USA, 15-19 May 2006.

7. Nansai, S.; Rojas, N.; Elara, M.R.; Sosa, R. Exploration of adaptive gait patterns with a reconfigurable linkage mechanism. In Proceedings of the 2013 IEEE/RSJ International Conference on Intelligent Robots and Systems, Tokyo, Japan, 3-7 November 2013.

8. Moubarak, P.; Ben-Tzvi, P. Modular and reconfigurable mobile robotics. Robot. Autom. Syst. 2012, 60, 1648-1663. [CrossRef]

9. Patil, M.; Abukhalil, T.; Sobh, T. Hardware Architecture Review of Swarm Robotics System: Self-Reconfigurability, Self-Reassembly, and Self-Replication. ISRN Robot. 2013, 2013. [CrossRef]

10. Kee, V.; Rojas, N.; Elara, M.R.; Sosa, R. Hinged-Tetro: A self-reconfigurable module for nested reconfiguration. In Proceedings of the 2014 IEEE/ASME International Conference on Advanced Intelligent Mechatronics, Besançon, France, 8-11 July 2014.

11. Prabakaran, V.; Elara, M.R.; Pathmakumar, T.; Nansai, S. hTetro: A tetris inspired shape shifting floor cleaning robot. In Proceedings of the 2017 IEEE International Conference on Robotics and Automation (ICRA), Singapore, 29 May-3 June 2017.

12. Prabakaran, V.; Elara, M.R.; Pathmakumar, T.; Nansai, S. Floor cleaning robot with reconfigurable mechanism. Autom. Constr. 2018, 91, 155-165. [CrossRef]

13. Prabakaran, V.; Mohan, R.E.; Sivanantham, V.; Pathmakumar, T.; Kumar, S.S. Tackling Area Coverage Problems in a Reconfigurable Floor Cleaning Robot Based on Polyomino Tiling Theory. Appl. Sci. 2018, 8, 342. [CrossRef]

14. Veerajagadheswar, P.; Elara, M.R.; Pathmakumar, T.; Ayyalusami, V. A Tiling-Theoretic Approach to Efficient Area Coverage in a Tetris-Inspired Floor Cleaning Robot. IEEE Access 2018, 6, 35260-35271. [CrossRef]

15. Twarock, R. The Architecture of Viral Capsids Based on Tiling Theory. J. Theor. Med. 2005, 6. [CrossRef]

16. Jho, C.W.; Lee, W.H. Video Puzzle Game Application of Polyomino Re-tiling. In Proceedings of the Embedded and Multimedia Computing Technology and Service, Lecture Notes in Electrical Engineering, Dordrecht, The Netherlands, 13 May 2012. 
17. Mardani, A.; Jusoh, A.; MD Nor, K.; Khalifah, Z.; Zakwan, N.; Valipour, A. Multiple criteria decision-making techniques and their applications-A review of the literature from 2000 to 2014. Econ. Res. Ekonomska Istraživanja 2015, 28, 516-571. [CrossRef]

18. Triantaphyllou, E.; Shu, B.; Sanchez, S.N.; Ray, T. Multi-Criteria Decision Making: An Operations Research Approach. Encyclopedia of Electrical and Electronics Engineering; Webster, J.G., Ed.; John Wiley \& Sons: New York, NY, USA, 1998; pp. 175-186.

19. Mardani, A.; Jusoh, A.; Zavadskas, E.K. Fuzzy multiple criteria decision-making techniques and applications-Two decades review from 1994 to 2014. Expert Syst. Appl. 2015, 42, 4126-4148. [CrossRef]

20. Zarghami, M.; Szidarovszky, F. Stochastic-fuzzy multi criteria decision making for robust water resources management. Stoch. Environ. Res. Risk Assess. 2009, 23, 329-339. [CrossRef]

21. Chen, T.-Y. The inclusion-based TOPSIS method with interval-valued intuitionistic fuzzy sets for multiple criteria group decision making. Appl. Soft Comput. 2015, 26, 57-73. [CrossRef]

22. Tzeng, G.-H.; Huang, J.-J. Multiple Attribute Decision Making, Methods and Applications; CRC Press: Boca Raton, FL, USA, 2011.

23. Roszkowska, E.; Wachowicz, T. Application of fuzzy TOPSIS to scoring the negotiation offers in ill-structured negotiation problems. Eur. J. Oper. Res. 2015, 242, 920-932. [CrossRef]

24. Landa-Torres, I.; Manjarres, D.; Bilbao, S.; Del Ser, J. Underwater Robot Task Planning Using Multi-Objective Meta-Heuristics. Sensors 2017, 17, 762. [CrossRef] [PubMed]

25. Devi, K. Extension of VIKOR method in intuitionistic fuzzy environment for robot selection. Expert Syst. Appl. 2011, 38, 14163-14168. [CrossRef]

26. Basilico, N.; Amigoni, F. Exploration strategies based on multi-criteria decision making for searching environments in rescue operations. Auton. Robots 2011, 31, 401. [CrossRef]

27. Befumo, A.; Lenchner, J. Extensions of Golombs Tromino Theorem. In Proceedings of the Fall Workshop in Computational Geometry, University of Connecticut, Storrs, CT, USA, 31 October-1 November 2014.

28. Golomb, S.W. Checker Boards and Polyominoes. Am. Math. Mon. 1954, 61, 675-682. [CrossRef]

29. Lester, C. Tilings with T and Skew Tetrominoes. Quercus Linfield J. Undergrad. Res. 2012, 1.

30. Nitica, V. The tilings of deficient squares by ribbon L-tetrominoes are diagonally cracked. arXiv 2017, arXiv:1701.00419.

31. Plonski, P.A.; Tokekar, P.; Isler, V. Energy-Efficient Path Planning for Solar-Powered Mobile Robots. In Experimental Robotics; Springer International Publishing: Heidelberg, Germany, 2013; pp. 717-731.

32. Patil, M.; Abukhalil, T.; Patel, S.; Sobh, T. UB robot swarm—Design, implementation, and power management. In Proceedings of the 12th IEEE International Conference on Control and Automation (ICCA), Kathmandu, Nepal, 1-3 June 2016.

33. Barili, A.; Ceresa, M.; Parisi, C. Energy-saving motion control for an autonomous mobile robot. In Proceedings of the IEEE International Symposium on Industrial Electronics, Dubrovnik, Croatia, 10-14 July 1995.

34. Duleba, I.; Sasiadek, J.Z. Nonholonomic motion planning based on Newton algorithm with energy optimization. IEEE Trans. Control Syst. Technol. 2003, 11, 355-363. [CrossRef]

(C) 2018 by the authors. Licensee MDPI, Basel, Switzerland. This article is an open access article distributed under the terms and conditions of the Creative Commons Attribution (CC BY) license (http://creativecommons.org/licenses/by/4.0/). 